To APPEAR IN ApJ

Preprint typeset using $\mathrm{LATEX}_{\mathrm{E}}$ style emulateapj v. 08/22/09

\title{
V819 TAU: A RARE WEAK-LINED T TAURI STAR WITH A WEAK INFRARED EXCESS
}

\author{
E. Furlan ${ }^{1,2}$, W. J. Forrest ${ }^{3}$, B. A. Sargent ${ }^{4}$, P. Manoj ${ }^{3}$, K. H. Kim ${ }^{3}$, Dan M. Watson ${ }^{3}$ \\ To appear in ApJ
}

\begin{abstract}
We use Spitzer data to infer that the small infrared excess of V819 Tau, a weak-lined T Tauri star in Taurus, is real and not attributable to a "companion" $10^{\prime \prime}$ to the south. We do not confirm the mid-infrared excess in HBC 427 and V410 X-ray 3, which are also non-accreting T Tauri stars in the same region; instead, for the former object, the excess arises from a red companion $9^{\prime \prime}$ to the east. A single-temperature blackbody fit to the continuum excess of V819 Tau implies a dust temperature of $143 \mathrm{~K}$; however, a better fit is achieved when the weak 10 and $20 \mu \mathrm{m}$ silicate emission features are also included. We infer a disk of sub- $\mu \mathrm{m}$ silicate grains between about 1 AU and several 100 AU with a constant surface density distribution. The mid-infrared excess of V819 Tau can be successfully modeled with dust composed mostly of small amorphous olivine grains at a temperature of $85 \mathrm{~K}$, and most of the excess emission is optically thin. The disk could still be primordial, but gas-poor and therefore short-lived, or already at the debris disk stage, which would make it one of the youngest debris disk systems known.
\end{abstract}

Subject headings: circumstellar matter — stars: formation — stars: individual (V819 Tau, HBC 427, V410 X-ray 3) — stars: pre-main sequence — infrared: stars

\section{INTRODUCTION}

The evolution and dissipation of protoplanetary disks has been an active area of research for the last few decades; especially the advent of sensitive near- and midinfrared observations has allowed us to explore inner disk regions (out to a few $\mathrm{AU}$ ), encompassing the radii where planets are thought to form. The role of planet formation in disk dissipation has been all but proven; while disks likely evolve from a flared, optically thick configuration to a flat, settled disk via grain growth and settling, it is not clear to what extent the eventual dissipation of the remaining dust and gas and the formation of planets are linked.

A key evolutionary stage in disk dissipation is the transition from the classical $\mathrm{T}$ Tauri stage, when a pre-main-sequence star is surrounded by an accreting disk, to the weak-lined $\mathrm{T}$ Tauri phase, when accretion ends and the disk disappears. This transitional stage is believed to be short ( $\lesssim 10^{5}$ years), since only few stars with vanishing infrared excesses have been observed (e.g., Skrutskie et al. 1990; Simon \& Prato 1995). The Infrared Spectrograph ${ }^{5}$ (IRS; Houck et al.|2004) on board the Spitzer Space Telescope (Werner et al. 2004) has provided new insights into the study of transitional disks. For example, in the nearby and well-studied Taurus star-forming region (Kenyon \& Hartmann 1995),

\footnotetext{
${ }^{1}$ Jet Propulsion Laboratory, California Institute of Technology, Mail Stop 264-767, 4800 Oak Grove Drive, Pasadena, CA 91109; Elise.Furlan@jpl.nasa.gov

${ }^{2}$ Spitzer Fellow

${ }^{3}$ Department of Physics and Astronomy, University of Rochester, Rochester, NY 14627; forrest@pas.rochester.edu, manoj@pas.rochester.edu, k khkim@pas.rochester.edu, dmw@pas.rochester.edu

${ }_{4}$ Space Telescope Science Institute, 3700 San Martin Drive, Baltimore, MD 21218; sargent@stsci.edu

5 The IRS was a collaborative venture between Cornell University and Ball Aerospace Corporation funded by NASA through the Jet Propulsion Laboratory and the Ames Research Center.
}

out of a total of $111 \mathrm{~T}$ Tauri stars, five objects were identified whose inner disk regions are cleared to different degrees, but outer, optically thick disks remain, delimited by a well-defined inner disk rim (D'Alessio et al. 2005); Calvet et al. 2005; Furlan et al. 2006; Espaillat et al. 2007). Planet formation, photoevaporation, or inner disk draining induced by the magneto-rotational instability may play a role in removing the inner disk Clarke. Gendrin, \& Sotomavor 2001; Quillen et al. 2004; Alexander \& Armitage 2007; Chiang \& Murray-Clav 2007), but also close binary companions can clear inner regions due to orbital resonances (Artymowicz \& Lubow 1994; Ireland \& Kraus 2008).

V819 Tau is a weak-lined T Tauri star with a spectral type of $\mathrm{K} 7$ in the Taurus star-forming region (Herbig \& Bell 1988). We presented its Spitzer IRS spectrum in Furlan et al. (2006) as part of the sample of Class III objects in Taurus, noting that it showed an infrared excess beyond about $12 \mu \mathrm{m}$ (see Figure 1). However, we could not confirm whether this excess was real due to the presence of a "companion" $10^{\prime \prime}$ to the south that is detected in 2MASS images (Skrutskie et al. 2006). Two other weak-lined T Tauri stars in Taurus, HBC 427 and V410 X-ray 3, with spectral types of K5 and M6, respectively (Steffen et al. 2001; Strom \& Strom 1994), were also introduced in Furlan et al. (2006) as objects with uncertain infrared excesses. While HBC 427 has a "companion" star about $15^{\prime \prime}$ to the southeast that is seen in 2MASS images, V410 X-ray 3 appears to be single. In the meantime, we obtained IRS peak-up images of V819 Tau and HBC 427, and we re-reduced the IRS spectra of all three objects. We do not confirm the mid-infrared excess in HBC 427 and V410 X-ray 3, but, together with Multiband Imaging Photometer for Spitzer (MIPS; Rieke et al. 2004) $24 \mu \mathrm{m}$ images from the Spitzer archive, we establish that the infrared excess is intrinsic to V819 Tau, and we apply simple models to derive the distribution of dust around this T Tauri star. 


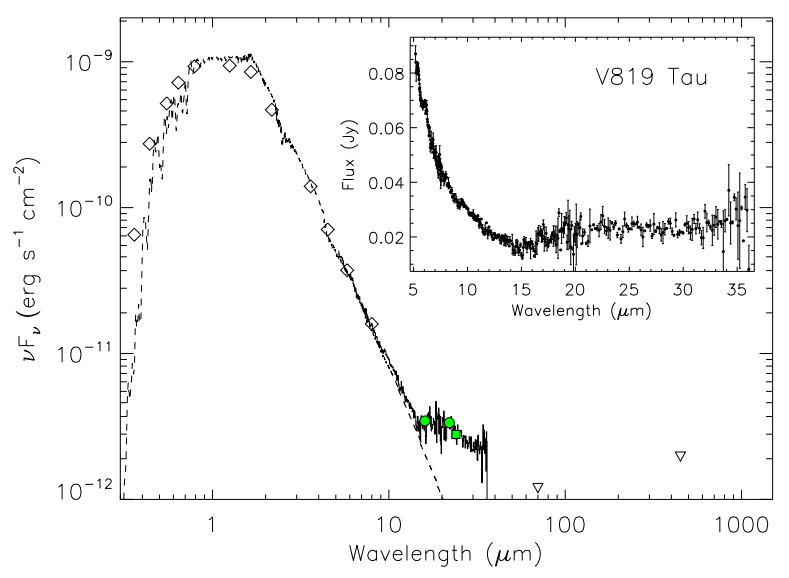

FIG. 1.- The spectral energy distribution of V819 Tau; the optical photometry is from Kenvon \& Hartmann (1995), $\mathrm{JHK}_{s}$ photometry from 2MASS (Skrutskie et al. 2006), IRAC fluxes from Luhman et al. (2006), and the $450 \mu \mathrm{m}$ upper limit from Andrews \& Williams (2005). The IRS spectrum (also shown with error bars in the figure inset), IRS peak-up fluxes, and MIPS fluxes are from this work. The data were dereddened using Mathis's reddening law for $R_{V}=3.1$ (Mathis 1990) and $A_{V}=1.7$ (Furlan et al. 2006). The stellar photosphere is a scaled NextGen model with $T_{\text {eff }}=4000 \mathrm{~K}$ and $\log (g)=3.5$ (see text for details).

\section{OBSERVATIONS}

\subsection{V819 Tau}

The IRS data of V819 Tau were taken on 2004 March 5 with the two low-resolution IRS modules (Short-Low [SL] and Long-Low [LL], 5.2-14 $\mu \mathrm{m}$ and 14-38 $\mu \mathrm{m}$, respectively, $\lambda / \Delta \lambda \sim 90)$ in staring mode, and the spectrum was extracted and calibrated as described in Furlan et al. (2006). The narrower SL slit (3.6" wide) was oriented roughly along the north-south direction, with the LL slit $\left(10.5^{\prime \prime}\right.$ wide) perpendicular to the SL slit (see a sketch of the slit positions in Figure 2). Since we extracted the object with a variable-width column that varies from a width of about 3 pixels to about 5 pixels from shortest to longest wavelengths in each module, the largest extraction radius in SL amounts to $4.5^{\prime \prime}$, while in LL this value is $\sim 13^{\prime \prime}$. These beams are just slightly larger than the diffraction-limited sizes of a point-source at 14 and $38 \mu \mathrm{m}$, respectively, observed with Spitzer. The companion star $10^{\prime \prime}$ to the south of V819 Tau is very faint at near-infrared wavelengths, with flux ratios relative to V819 Tau of $0.04-0.03$ from $J$ to $K_{s}$; if it were a very red object, it could have contaminated some of the emission in LL, but not in SL.

To determine the mid-infrared flux of the "companion", we obtained IRS red $(16 \mu \mathrm{m})$ and blue $(22$ $\mu \mathrm{m}$ ) peak-up images on 2006 March 7 (Spitzer AORID 16269312). We dithered the target in two positions displaced by $9^{\prime \prime}$ from the center of the peak-up field of view; at each position we integrated for 6 seconds. For the red peak-up images, we performed this sequence twice. By subtracting two such nod pairs from each other, we removed any background emission; aperture photometry with a radius of 3 pixels and a sky annulus between 18 and 20 pixels yielded 16 and $22 \mu \mathrm{m}$ fluxes of 18.4 \pm 0.2 and $24.6 \pm 0.2 \mathrm{mJy}$, respectively, for V819 Tau. These flux values include aperture correction factors of 1.418 and 1.561 for the blue and red peak-up, respectively, and they were derived assuming a spectral shape of $\nu F_{\nu}=$ constant for the source spectrum (see the docu-
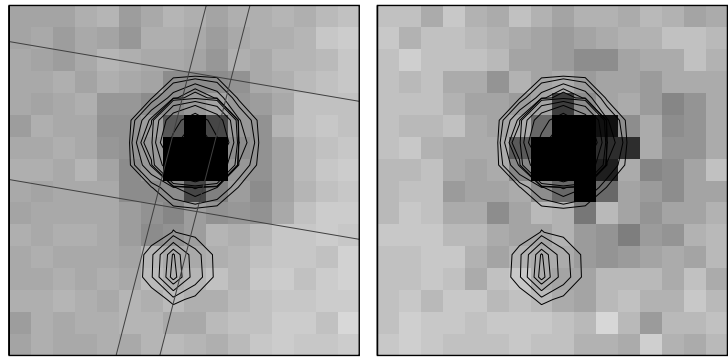

FIG. 2.- IRS peak-up images (left: $16 \mu \mathrm{m}$; right: $22 \mu \mathrm{m}$ ) of V819 Tau, with superposed contours of the 2MASS $K_{s}$ image. North is up, east is to the left; the plate scale amounts to $1{ }^{\prime \prime} 8 /$ pixel, and the field of view is $29^{\prime \prime} \times 29^{\prime \prime}$. The SL and LL slit positions are also sketched in the $16 \mu \mathrm{m}$ image; the SL slit is the narrower one running roughly north-south.

ment pu_fluxcal.txt found on the IRS page of the Spitzer Science Center's website).

Figure 2 shows the 16 and $22 \mu \mathrm{m}$ peak-up images of V819 Tau; each represents the sum of two peak-up exposures and was rotated such that north is up and east is to the left. Superposed on Figure 2 are the contours of the 2MASS $K_{s}$ image. While the southern "companion" is clearly present in $2 \mathrm{MASS}$, it is not detected at 16 or $22 \mu \mathrm{m}$. We derived upper limits for its flux from the rms in $3 \times 3$ (for the blue peak-up) and $5 \times 5$ (for the red peakup) pixel boxes centered at its position in the co-added image pairs. We thus obtained 16 and $22 \mu \mathrm{m} \mathrm{5- \sigma}$ upper limits of 0.8 and $2.0 \mathrm{mJy}$, respectively.

We also retrieved the MIPS 24 and $70 \mu \mathrm{m}$ post-bcd, mosaicked images from the Spitzer archive (program 3584, PI D. Padgett; Spitzer AORID 11229696). By using a circular aperture with a radius of 14 pixels and a sky annulus between 16 and 20 pixels, and by applying an aperture correction factor of 1.082 , we measured a flux of $22.3 \pm 1.3 \mathrm{mJy}$ at $24 \mu \mathrm{m}$ for V819 Tau. At $70 \mu \mathrm{m}$, we used the filtered bcd image from the Spitzer archive and derived a 5- $\sigma$ upper limit of $30 \mathrm{mJy}$ from the rms in a $13 \times 13$ pixel box centered at the position of V819 Tau. These fluxes assume a spectral shape of $\nu F_{\nu} \propto \nu^{3}$; corrections for different spectral shapes are minor, e.g., $4 \%$ at $24 \mu \mathrm{m}$ and $9 \%$ at $70 \mu \mathrm{m}$ for $\nu F_{\nu}=$ constant (see MIPS Data Handbook).

Thus, the imaging data confirms that the IRS spectrum of V819 Tau is not contaminated at any significant level by the source that is seen to its south at nearIR wavelengths. We will therefore consider the longwavelength emission as coming only from the disk around V819 Tau.

\section{2. $H B C 427$}

Similar to V819 Tau, the IRS data of HBC 427 were taken on 2004 February 27 with the SL and LL modules in staring mode and reduced in the same manner. The slits were oriented similarly to V819 Tau (see Figure 3). In 2MASS, only two sources are detected; the southern "companion" is about half as bright as HBC 427 at $K_{s}$, but $15^{\prime \prime}$ from it $(\sim 8$ " from the nearest edge of the LL slit), making it unlikely to contribute substantially to the LL flux. However, after we obtained IRS red and blue peak-up images of HBC 427 on 2006 March 8 (Spitzer AORID 16270336), we discovered an infraredbright companion of HBC 427 that does not show up in 2MASS images. It lies $\sim 9^{\prime \prime}$ to the east of HBC 427 

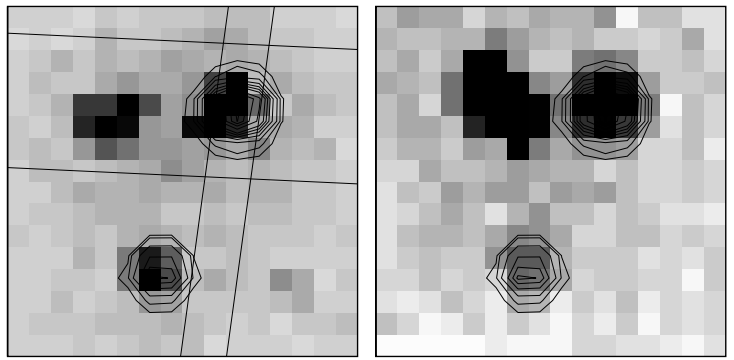

FIg. 3.- As Figure 2 but for HBC 427.

and is the brightest object of the three at $22 \mu \mathrm{m}$. This object, HBC $427 / 1$, was actually already identified by Massarotti et al. (2005), who imaged it at $1.025 \mu \mathrm{m}$ and retrieved it from the USNO-B1.0 catalog. Our LL observation represents the combined flux of $\mathrm{HBC} 427$ and HBC $427 / 1$; in particular starting at about $20 \mu \mathrm{m}$, the latter source dominates the emission.

In order to measure the peak-up fluxes of HBC 427 and HBC $427 / 1$, we performed aperture photometry. Even though our observing sequence was the same as for the peak-up observations of V819 Tau, we could not subtract the background by subtracting the nod pairs, since the field was too crowded. Thus, the only sky subtraction was performed during aperture photometry, where we used an aperture radius of 2 pixels and a sky annulus between 11-12 and 13-14 pixels. By applying aperture correction factors of 1.661 and 1.885 for the blue and red peak-up, respectively, and assuming a spectral shape of $\nu F_{\nu}=$ constant, we derived $16 \mu \mathrm{m}$ fluxes of $11.0 \pm 0.2$ mJy and $7.2 \pm 0.2$ mJy for HBC 427 and HBC $427 / 1$, respectively, and $22 \mu \mathrm{m}$ fluxes of $7.0 \pm 0.2 \mathrm{mJy}$ and 14.4 \pm 0.3 mJy for HBC 427 and HBC $427 / 1$, respectively.

We also measured the fluxes of these two objects in the MIPS $24 \mu \mathrm{m}$ post-bcd, mosaicked image from the Spitzer archive (program 173, PI N. Evans; Spitzer AORID 5683200). Applying a PSF fit using the star HD 31305, which appears in the same image, as PSF calibrator, yielded $24 \mu \mathrm{m}$ fluxes of $5.0 \pm 1.3 \mathrm{mJy}$ and $17.8 \pm 1.2$ mJy for HBC 427 and HBC $427 / 1$, respectively.

We obtained an IRS spectrum of HBC $427 / 1$ on 2006 March 15 (Spitzer AORID 16262400) using SL and LL in staring mode. While we were able to extract and calibrate the SL spectrum for HBC 427/1, the LL spectrum is again a combination of the flux of HBC 427/1 and HBC 427. Since this LL spectrum has better signalto-noise than the one obtained earlier due to longer integration times, we use this spectrum in the following plot. Figure 4 shows the IRS spectra of both HBC 427 and HBC $427 / 1$, as well as the photometry we obtained from the peak-up and MIPS images. A blackbody fit to the SL spectrum demonstrates that the 16, 22, and 24 $\mu \mathrm{m}$ fluxes of HBC 427 are essentially photospheric; the infrared excess is solely attributable to HBC 427/1.

\subsection{V410 X-ray 3}

The IRS data of V410 X-ray 3 were taken on 2004 Feb 7 , also using SL and LL in starting mode. Since this object is very faint in the first order of LL $(20-36 \mu \mathrm{m})$, we first subtracted sky emission by using the off-order observation, then extracted its $20-36 \mu \mathrm{m}$ spectrum while also fitting a first order polynomial to the background emission in the slit. This procedure allowed for more

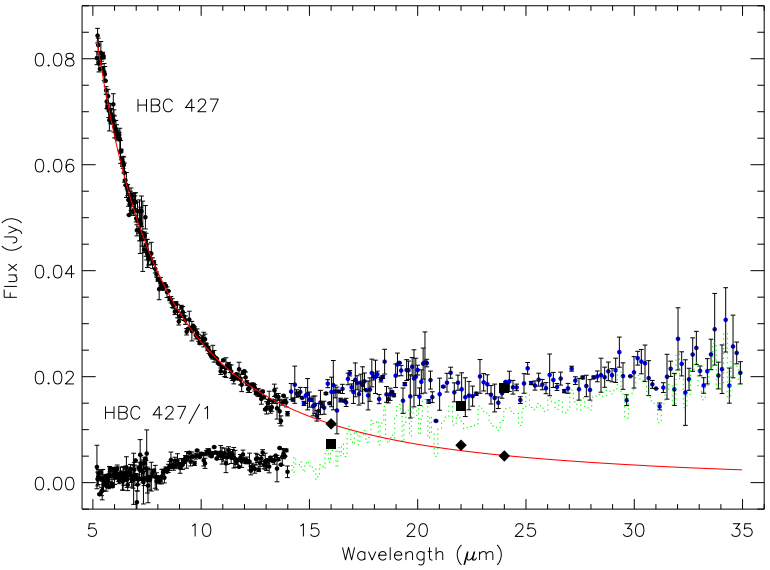

FIG. 4.- IRS spectra of HBC 427 and HBC $427 / 1$; the LL spectrum represent emission from both objects. The filled diamonds and squares are the peak-up and MIPS $24 \mu \mathrm{m}$ fluxes of HBC 427 and HBC $427 / 1$, respectively. The red solid line shows a blackbody fit to the SL spectrum of HBC $427(\mathrm{~T}=4310 \mathrm{~K}, \Omega=2.65 \times$ $\left.10^{-19} \mathrm{sr}\right)$. The green dotted line is an estimate of the LL spectrum of HBC $427 / 1$, obtained by subtracting the blackbody fit from the combined LL spectrum. The data were dereddened using Mathis's reddening law for $R_{V}=3.1$ (Mathis 1990) and $A_{V}=0.5$ (Furlan et al. 2006).

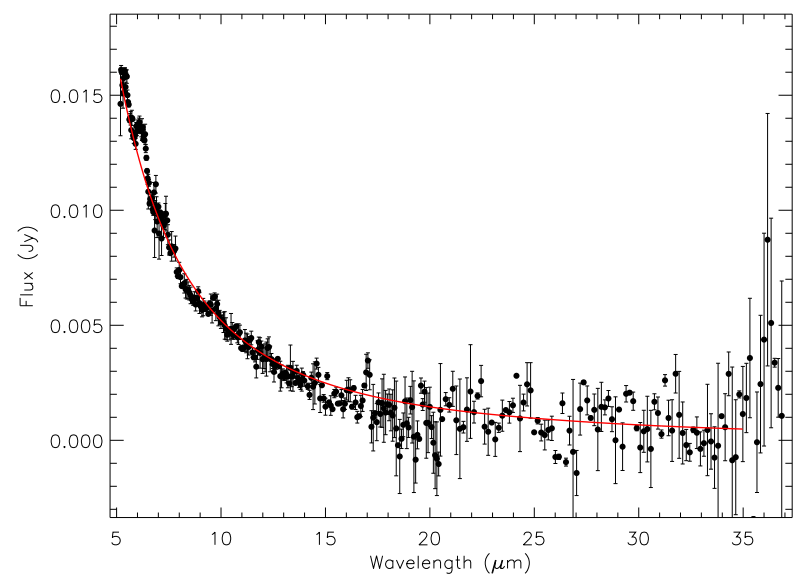

FIG. 5.- IRS spectrum of V410 X-ray 3 and a blackbody fit to the SL spectrum with $\mathrm{T}=3520 \mathrm{~K}$ and $\Omega=6.62 \times 10^{-20}$ sr (red, solid line). The data were dereddened using Mathis's reddening law for $R_{V}=3.1$ (Mathis 1990) and $A_{V}=1.5$ (Kraus et al. 2006).

accurate background subtraction. We compared reductions done with basic calibrated data products from the Spitzer Science Center's S11, S13.2, and S18.7 pipeline versions, and the differences were minimal. As a result of the improved background subtraction, we do not confirm the tentative excess seen in Furlan et al. (2006); the IRS spectrum seems consistent with photospheric emission alone (see Figure 5).

\section{SPECTRAL ENERGY DISTRIBUTIONS}

Skrutskie et al. (1990) stated that V819 Tau has a spectral energy distribution (SED) that identifies it as a "transitional" disk, i.e., a disk with an optically thin inner region and an optically thick outer disk. Beckwith et al. (1990) detected millimeter-continuum emission from V819 Tau and inferred a disk mass of 0.032 $M_{\odot}$, implying that its outer disk is fairly massive. However, the far-IR SED of Skrutskie et al. (1990) had to rely on IRAS data, which are not reliable for such a faint 
source: according to Weaver \& Jones (1992), the IRAS 25 and $60 \mu \mathrm{m}$ fluxes of V819 Tau are both $100 \mathrm{mJy}$, while our data shows that the flux at $30 \mu \mathrm{m}$ amounts to $20 \mathrm{mJy}$. In addition, later measurements by Osterloh \& Beckwith (1995) and Duvert et al. (2000) only determined an upper limit to the $1.3 \mathrm{~mm}$ flux of 9 and $2.5 \mathrm{mJy}$, respectively, and Andrews \& Williams (2005) could also only give upper limits to the 450 and $850 \mu \mathrm{m}$ fluxes (317 and 9 mJy, respectively); the latter authors determined a disk mass of less than $0.0004 M_{\odot}$. Thus, it seems that the outer disk is not very massive.

Since the more recent measurements are much more precise, they should be favored when interpreting V819 Tau. Given that the excess emission from this T Tauri star is weak, earlier data with less sensitive instruments are more uncertain. The object $10^{\prime \prime}$ to the south of V819 Tau is even fainter; its low infrared flux values support the suggestion of Woitas et al. (2001) that it is likely a background star due to its low luminosity and its inferred age that places it well below the main sequence when plotted with evolutionary tracks.

Opposite to what we found for V819 Tau, the new Spitzer data of HBC 427 show that the infrared excess seen in the long-wavelength part of the IRS spectrum is attributable to a nearby companion, HBC 427/1. Even though Massarotti et al. (2005) suggest it could be a hot background star based on its optical to near-IR colors, the infrared excess indicates that it is an object surrounded by dust. We estimated the emission of $\mathrm{HBC}$ $427 / 1$ beyond $14 \mu \mathrm{m}$ by subtracting a blackbody fit to the SL spectrum of HBC 427 from the LL spectrum (see Figure 4); the 16, 22, and $24 \mu \mathrm{m}$ photometry of HBC $427 / 1$ agrees reasonably well with this estimated spectrum. The infrared excess consists of continuum emission, as well as optically thin radiation from silicate dust grains, whose signatures are broad 10 and $20 \mu \mathrm{m}$ emission features, similar to what is seen in protoplanetary disks (e.g., Furlan et al. 2006). However, without any additional data, we cannot conclusively determine the nature of this object.

The very low-mass star V410 X-ray 3 is fainter than V819 Tau or HBC 427; only a careful new reduction of its IRS spectrum showed that it does not have an infrared excess. A blackbody fit to the $5-14 \mu \mathrm{m}$ spectrum also matches the 15-35 $\mu \mathrm{m}$ fluxes (see Figure 5). However, the spectrum beyond $20 \mu \mathrm{m}$ is quite noisy; we can therefore not exclude excesses smaller than about $1 \mathrm{mJy}$ in this wavelength range. Luhman et al. (2009) find that a very small excess of $\sim 0.4$ mag may be present in MIPS $24 \mu \mathrm{m}$ data, which is smaller than our upper limit of $\sim 1$ mag for an excess at this wavelength. More sensitive observations at longer wavelengths are necessary to confirm this small excess; given the current data, we consider V410 X-ray 3 as diskless.

\section{THE IRS SPECTRUM AND MODEL FITS OF V819 TAU}

Our IRS spectrum confirms the transitional disk nature of V819 Tau, but it reveals that the mid-infrared excess is much smaller than previously thought (see Figure 11); it sets in at about $8 \mu \mathrm{m}$, and $F_{\nu}$ is roughly constant with wavelength between 23 and $33 \mu \mathrm{m}$. The very small infrared excess $\left(L_{I R} / L_{b o l} \sim 10^{-3}\right)$ makes V819 Tau the most advanced transitional disk in the Taurus sample observed to date. Also, as opposed to the other tran-

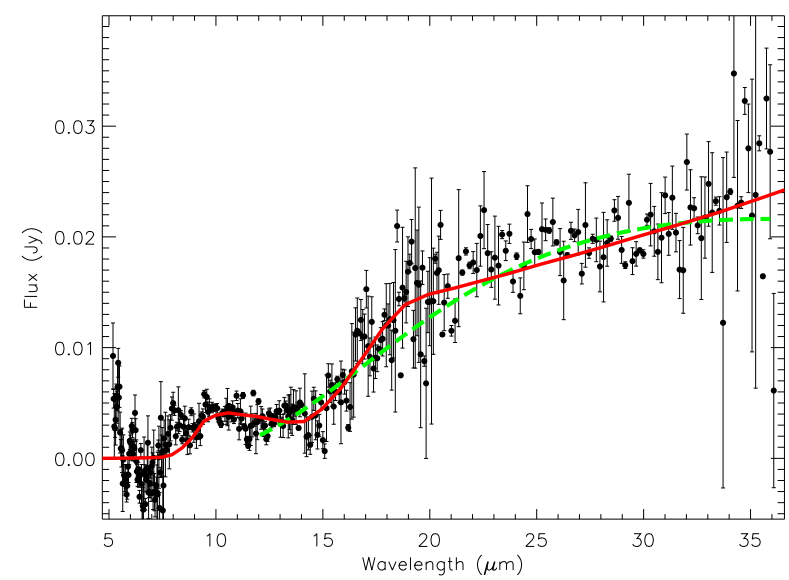

FIG. 6.- Photosphere-subtracted IRS spectrum (see text for details) and fits to the infrared excess: a blackbody fit from 12 to $36 \mu \mathrm{m}$ (dashed green line), yielding a temperature of $143 \mathrm{~K}$ and a solid angle of the emitting area of $3.90 \times 10^{-16} \mathrm{sr}$, and an optically thin model fit (solid red line). The latter model is one of several very similar fits that use amorphous olivines with grains ranging in size between 0.1 and $1 \mu \mathrm{m}$ (up to 15 discrete sizes), distributed between 1 and several hundred AU; the surface density is constant with respect to radius and amounts to $\sim 4 \times 10^{-7} \mathrm{~g} \mathrm{~cm}^{-2}$ for all grains.

sitional disks, whose inner holes are delimited by a disk wall that gives rise to a steep continuum slope beyond 13 $\mu \mathrm{m}$, the spectral index between 13 and $25 \mu \mathrm{m}$ for $\mathrm{V} 819$ Tau is more negative, in a range typical for settled disks (Furlan et al. 2006).

V819 Tau is a K7 star (Walter \& Kuhi 1981), which suggests $T_{\text {eff }}=4060 \mathrm{~K}$ (Kenvon \& Hartmann 1995); combined with a luminosity of $0.81 L_{\odot}(\mathrm{KH} 95)$, we derive a stellar radius of $1.8 R_{\odot}$. Using evolutionary tracks computed by Baraffe et al. (1998), we infer a stellar mass of $0.8 M_{\odot}$ (as well as an age of $2 \mathrm{Myr}$ ), which indicates that the surface gravity is of the order $\log (g) \sim 3.5-4.0$. Thus, for the photosphere we adopt one of the NextGen photospheric models (Allard. Hauschildt, \& Schweitzer 2000) with $T_{\text {eff }}=4000 \mathrm{~K}, \log (g)=3.5$, scaled by assuming a stellar radius of $2 R_{\odot}$ and a distance of $140 \mathrm{pc}$ (Bertout et al. 1999).

The photosphere-subtracted IRS spectrum is shown in Figure 6. A single-temperature blackbody fit from 12 to $36 \mu \mathrm{m}$ yields a blackbody temperature of $143 \mathrm{~K}$. If the excess emission were optically thick, generated by a solid blackbody surface, this temperature would imply an inner disk radius of 6.8 AU. However, the low excess flux at longer wavelengths is not typical for optically thick emission. Assuming optically thin emission from gray grains in radiative equilibrium whose absorption cross section equals their geometric cross section (i.e, large, blackbody grains), the inner disk radius would lie at 3.4 AU. More realistically, the absorption efficiency varies with wavelength, as is the case for smaller grains (e.g., Dorschner et al. 1995). We actually detect the signatures of small, amorphous grains of silicate composition ("amorphous silicates") in an optically thin medium as weak, broad features centered at 10 and $20 \mu \mathrm{m}$ (e.g., Jäger et al. 1994) in the photosphere-subtracted spectrum. If the dust grains are small (sub- $\mu \mathrm{m})$, their absorption efficiency in the infrared is less than at shorter wavelengths, leading to so-called superheating, which would result in a grain being at a somewhat larger distance from 
TABLE 1

Dust Components of V819 Tau

\begin{tabular}{lll}
\hline \hline \multicolumn{1}{c}{ Dust component } & \multicolumn{1}{c}{$\mathrm{T}[\mathrm{K}]$} & \multicolumn{1}{c}{ Mass [lunar masses] } \\
\hline small amorphous olivine $^{a}$ & 345 & $(2.0 \pm 1.4) \times 10^{-6}$ \\
small amorphous olivine $^{a}$ & 85 & $(9.9 \pm 0.8) \times 10^{-3}$ \\
large amorphous pyroxene $^{b}$ & 85 & $(3.1 \pm 0.8) \times 10^{-3}$ \\
forsterite $^{c}$ & 85 & $(1.2 \pm 0.5) \times 10^{-3}$ \\
blackbody $^{d}$ & $345 \mathrm{~K}$ & $(4.5 \pm 0.7) \times 10^{-18} \mathrm{sr}$ \\
\hline
\end{tabular}

Note. - (a) Optical constants from Dorschner et al. (1995)); (b) optical constants from Jäger et al. (1994); (c) optical constants from Sogawa et al. (2006); (d) the quantity listed in the third column is the solid angle.

the star than a blackbody at the same temperature.

The lack of substantial excess emission below about $8 \mu \mathrm{m}$ suggests that the inner disk regions are cleared of small dust grains. In order to derive the disk structure of V819 Tau, we applied an optically thin model fit to the infrared excess emission $(8-34 \mu \mathrm{m})$. We adopted the optical constants of "astronomical" silicates from Draine \& Lee (1984) and chose different grain sizes with various surface density distributions (which act as a scaling factor for the emission) to match the observed excess. The grain size distribution was assumed to vary as a power law with an index of -3.5. At each distance from the star, we calculated the equilibrium temperature for the average grain size, and then integrated the optically thin emission from all grains over a certain radius range to achieve a good fit.

A range of model parameters results in fits that reproduce the weak 10 and $20 \mu \mathrm{m}$ silicate emission features and rising continuum seen in the photosphere-subtracted spectrum: minimum grain sizes from 0.1 to $0.5 \mu \mathrm{m}$, maximum grain sizes of 0.5 or $1 \mu \mathrm{m}$, an inner disk radius of $1 \mathrm{AU}$, and outer disk radii from 300 to 800 AU. Several models lie within reduced $\chi^{2}\left(\chi_{\nu}^{2}\right)$ values of 0.3 from the best-fit model $\left(\chi_{\nu}^{2}=19.1\right)$, which is shown in Figure 6, These models are basically indistinguishable except for slight differences beyond $30 \mu \mathrm{m}$, where the flux is most sensitive to the outer disk radius; at $70 \mu \mathrm{m}$, they all lie at or below the upper limit derived from MIPS data. A surface density constant in radius is necessary to reproduce the slowly rising 20-35 $\mu \mathrm{m}$ excess, as is an outer radius of several $100 \mathrm{AU}$; if the disk were smaller than $\sim 100 \mathrm{AU}$, the model would still fit the excess out to $20 \mu \mathrm{m}$, but increasingly underpredict the flux at longer wavelengths. An inner radius of $0.5 \mathrm{AU}$ instead of $1 \mathrm{AU}$ results in an increase in $\chi_{\nu}^{2}$ by about 1 . Summarizing, we require sub- $\mu \mathrm{m}$ grains distributed uniformly between $\sim 1 \mathrm{AU}$ and at least $100 \mathrm{AU}$ to reproduce the infrared excess of V819 Tau. The mass of optically thin dust from our models amounts to up to $2.4 \times 10^{26} \mathrm{~g}$, or 3.2 lunar masses, for the largest disk sizes, and about $4.6 \times 10^{25}$ g, or 0.6 lunar masses, for a disk of $\sim 400$ AU radius. The optical depth of the emission by all grains is only $~$ $10^{-4}-10^{-3}$ over the infrared spectral range.

In order to gauge the dust composition of the V819 Tau disk, we applied a model to the photospheresubtracted spectrum beyond $7.7 \mu \mathrm{m}$ following the methods of Sargent et al. (2006, 2009a, b) $)$. The best $\chi_{\nu}^{2}$ value of 14.7 is achieved by using a warm dust component at $345 \mathrm{~K}$ and a cold one at $85 \mathrm{~K}$. The warm component includes a blackbody, which represents continuum emis-

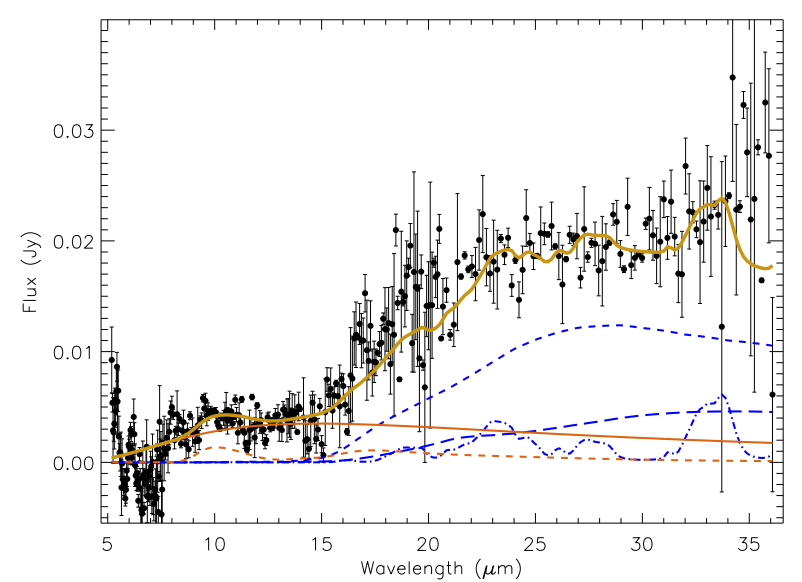

FIG. 7.- IRS spectrum as in Fig. 6 but with an optically thin model fit aimed at deriving the dust composition. The orange lines represent the warm dust $(345 \mathrm{~K})$, the blue lines the cold dust $(85 \mathrm{~K})$; the dust components required for the fit are small amorphous olivine (dashed lines), large amorphous pyroxene (longdashed lines), forsterite (dash-dotted line), and a blackbody component (solid, thin line). The thick line is the sum of all components.

sion from an optically thick medium or featureless emission from grains in an optically thin medium, and small amorphous silicates of olivine composition ("amorphous olivine"). The cold component is dominated by small amorphous olivine, with contributions of large amorphous silicates of pyroxene composition ("amorphous pyroxene") and forsterite (see Table 1 and Figure 7). These model components represent a best fit to the data and were narrowed down from a larger sample of dust components that included small and large amorphous silicates, enstatite, forsterite, and silica, which are typically found in protoplanetary disks (see Sargent et al. (2009b) for details). The uncertainties listed in Table 1 actually amount to a few standard deviations (see Sargent et al. 2009b); combined with the fact that a blackbody cannot fit the data well (see Figure 6), the identification of amorphous silicates in our data is quite robust. However, the detection of forsterite is rather tentative, since the spectrum is more noisy beyond $20 \mu \mathrm{m}$.

The dust mass is dominated by cold, sub- $\mu \mathrm{m}$ amorphous olivine grains, which are typical for the interstellar medium. The presence of some large $(5 \mu \mathrm{m}$ radius, $60 \%$ vacuum porous) amorphous pyroxene grains, and possibly also forsterite, suggests that some dust processing has taken place. Assuming a distribution of dust between 1 AU and several tens of AU, the optical depth amounts to $\lesssim 10^{-3}$. Thus, the dust we observe in V819 Tau is dominated by silicates in an optically thin medium.

\section{DISCUSSION AND CONCLUSIONS}

Of the three Class III objects in Taurus that showed tentative mid-infrared excess emission in Furlan et al. (2006), we could confirm an infrared excess only in V819 Tau. While V819 Tau is a single star, HBC 427 and V410 X-ray 3 are close binaries: HBC 427 is a spectroscopic binary with a semi-major axis of about $0.03^{\prime \prime}$, comprised of a K5 and an M2 star (Steffen et al. 2001); V410 Xray 3 consists of an M6 and an M7.7 star separated by $\sim 0.05^{\prime \prime}$ (Kraus et al. 2006). Neither object shows signs of accretion (Strom \& Strom 1994; Kenyon et al. 1998; Mohanty et al. 2005), and therefore both dust and 
gas have already dissipated in their inner disks. HBC 427 is also not detected at sub-millimeter wavelengths, implying an upper limit for the disk mass of 0.0007 $M_{\odot}($ Andrews \& Williams 2005). It is likely that interaction between the binary and the disk in these systems is the cause for the absence of disk material (Jensen et al. 1994).

V819 Tau is also not accreting any more; its $\mathrm{H} \alpha$ $10 \%$ width amounts to $166 \mathrm{~km} \mathrm{~s}^{-1}$ (Nguven et al. 2009), which is considerably less than the lower limit of 270 $\mathrm{km} \mathrm{s}^{-1}$ that characterizes accretors (White \& Basri 2003). Also the low $\mathrm{H} \alpha$ equivalent width of 1.7-3.2 $\AA$ (Strom \& Strom 1994; Kenyon et al. 1998) confirms its nature as a weak-lined $\mathrm{T}$ Tauri star. White \& Ghez (2001) determined an upper limit of $1.4 \times 10^{-9} M_{\odot} \mathrm{yr}^{-1}$ for the mass accretion rate based on the lack of $U$-band excess. No $\mathrm{H}_{2}$ emission from warm inner disk regions was detected (Barv. Weintraub, \& Kastner 2003), implying that both the gas and dust have been removed there. Our data indicate that there are no small, warm dust grains within approximately 1 AU from the star.

One process that can generate cleared

inner disk regions is photoevaporation
(Clarke, Gendrin, \& Sotomayor 2001); it sets in once the mass accretion rate has dropped to levels below a few $10^{-10} M_{\odot} \mathrm{yr}^{-1}$, and it acts by eroding the disk in a photoevaporative flow beyond a certain radius, which, for V819 Tau, amounts to 7.1 AU. This value is larger than the inner radius of our best-fit optically thin disk models, but it is remarkably close to the value of $6.8 \mathrm{AU}$ we estimated by assuming the excess emission to arise from an optically thick, blackbody surface. However, it seems that the dust emission is optically thin, given the presence of 10 and $20 \mu \mathrm{m}$ silicate emission features and the low infrared excess luminosity $\left(L_{I R} / L_{b o l} \sim\right.$ $\left.10^{-3}\right)$. Moreover, our models require small dust grains at distances of at least $\sim 1$ AU from the star. Thus, photoevaporation cannot explain the inner disk clearing of V819 Tau; a change in grain opacity caused by grain growth to sizes well beyond $1 \mu \mathrm{m}$ in this region, or the gravitational influence of another body, such as a planet, are possible explanations for the inner disk clearing.

The question also arises whether the material we detect around V819 Tau is primordial or already secondgeneration dust. Detection of gas emission from the inner disk would support the primordial nature of the disk; so far, the absence of accretion signatures and $\mathrm{H}_{2}$ emission from the inner disk suggest that the gas has already dissipated. Without the gas, dust particles would be subject to collisions, Poynting-Robertson (PR) drag, radiation pressure, and corpuscular stellar wind pressure (e.g., Wyatt et al. 1999; Chen et al. 2006), which, in the region from $\sim 1$ to $100 \mathrm{AU}$ from V819 Tau, would limit the lifetime of sub- $\mu \mathrm{m}$ grains to $\lesssim 10^{5}$ years.

Therefore, in the absence of gas, dust would have to be continuously replenished, since the age of V819 Tau is about 2 Myr. V819 Tau would be one of the youngest debris disk systems; its infrared excess would lie on the higher side of what is usually measured in debris disks (e.g., Chen et al. 2006), but still within the observed range. Collisions among planetesimals, as are thought to occur in debris disks, typically produce larger grains
( $\gtrsim 10 \mu \mathrm{m}$ ), since the mid-infrared spectra of the majority of debris disks are featureless (Chen et al. 2006; Carpenter et al. 2009). The fact that small, amorphous silicate grains dominate the optically thin emission of V819 Tau, with just a minor possible presence of crystalline silicates, suggests that the dust we observe consists of mostly pristine material. Colliding or evaporating comet nuclei, which likely form in the outer disk regions and therefore incorporate more unprocessed silicates into ice, could be the source of this pristine dust.

It is also possible that we are just observing "true" primordial, unprocessed dust from large disk radii; this would imply that we are witnessing a rare stage in the life of a protoplanetary disk, when the gas has already been mostly removed from the inner disk, and the remaining dust is dissipated on a timescale of $10^{5}$ years or less. Then, not only could the disk structure of V819 Tau be described as transitional due to the lack of infrared excess below $\sim 8 \mu \mathrm{m}$ and the clear presence of such an excess beyond $12 \mu \mathrm{m}$, but also the evolutionary stage of this object could be considered transitional, i.e., having just transitioned from a primordial, optically thick disk to an optically thin one. Even though a few percent of the T Tauri population in Taurus can be considered as transitional (defined as disks with substantial inner disk clearings and thus low near-infrared excesses; Furlan et al. 2009), V819 Tau would stand out by its very low infrared excess. It would be the only object in Taurus observed so far at an advanced transitional stage, when the disk material is already optically thin. Given the small amount of dust left in the disk, planet formation would have to be already completed in this disk; this conclusion would also apply if the dust were secondgeneration and therefore the result of dynamically perturbed, remnant planetary building blocks.

We thank the referee for helpful comments that led us to expand and improve this paper. This work is based on observations made with the Spitzer Space Telescope, which is operated by the Jet Propulsion Laboratory (JPL), California Institute of Technology (Caltech), under NASA contract 1407. Support for this work was provided by NASA through contract number 1257184 issued by JPL/Caltech. E.F. was partly supported by a NASA Postdoctoral Program Fellowship, administered by Oak Ridge Associated Universities through a contract with NASA, and partly supported by NASA through the Spitzer Space Telescope Fellowship Program, through a contract issued by JPL/Caltech under a contract with NASA. This publication makes use of data products from the Two Micron All Sky Survey, which is a joint project of the University of Massachusetts and the Infrared Processing and Analysis Center/California Institute of Technology, funded by NASA and the NSF. It has also made use of the SIMBAD and VizieR databases, operated at CDS (Strasbourg, France), NASA's Astrophysics Data System Abstract Service, and of the NASA/ IPAC Infrared Science Archive operated by JPL, Caltech, under contract with NASA.

Facilities: Spitzer(IRS) 


\section{REFERENCES}

Alexander, R. D. \& Armitage, P. J. 2007, MNRAS, 375, 500

Allard, F., Hauschildt, P. H., \& Schweitzer, A. 2000, ApJ, 539, 366

Andrews, S. M., \& Williams, J. P. 2005, ApJ, 631, 1134

Artymowicz, P., \& Lubow, S. H. 1994, ApJ, 421, 651

Baraffe, I., Chabrier, G., Allard, F., \& Hauschildt, P. H. 1998, A\&A, 337, 403

Bary, J. S., Weintraub, D. A., \& Kastner, J. H. 2003, ApJ, 586, 1136

Beckwith, S. V. W., Sargent, A. I., Chini, R. S., \& Güsten, R. 1990, AJ, 99, 924

Bertout, C., Robichon, N., \& Arenou, F. 1999, A\&A, 352, 574

Calvet, N., et al. 2005, ApJ, 630, L185

Carpenter, J. M., Bouwman, J., Mamajek, E. E., Meyer, M. R., Hillenbrand, L. A., Backman, D. E., Henning, Th., Hines, D. C., et al. 2009, ApJS, 181, 197

Chen, C. H., Sargent, B. A., Bohac, C., Kim, K. H., Leibensperger, E., Jura, M., Najita, J., Forrest, W. J., et al. 2006, ApJS, 166, 351

Chiang, E., \& Murray-Clay, R. 2007, Nature Physics, 3, 604

Clarke, C. J., Gendrin, A., \& Sotomayor, M. 2001, MNRAS, 328, 485

D'Alessio, P., et al. 2005, ApJ, 621, 461

Dorschner, J., Begemann, B., Henning, Th., Jäger, C., \& Mutschke, H. 1995, A\&A, 300, 503

Draine, B. T., \& Lee, H. M. 1984, ApJ, 285, 89

Duvert, G., Guilloteau, S., Ménard, F., Simon, M., \& Dutrey, A. 2000, A\&A, 355, 165

Espaillat, C., Calvet, N., D’Alessio, P., Hernández, J., Qi, C., Hartmann, L., Furlan, E., \& Watson, D. M. 2007, ApJ, 670, L135

Furlan, E., Hartmann, L., Calvet, N., D'Alessio, P., Franco-Hernández, R., Forrest, W. J., Watson, D. M., Uchida, K. I., et al. 2006, ApJS, 165, 568

Furlan, E., Watson, D. M., McClure, M. K., Manoj, P., Espaillat, C., D'Alessio, P., Calvet, N., Kim, K. H., et al. 2009, ApJ, 703, 1964

Herbig, G. H., \& Bell, K. R. 1988, Lick Observatory Bulletin, Santa Cruz: Lick Observatory, 1988

Houck, J. R., et al. 2004, ApJS, 154, 18

Ireland, M. J., \& Kraus, A. L. 2008, ApJ, 678, L59

Jäger, C., Mutschke, H., Begemann, B., Dorschner, J., \& Henning, Th. 1994, A\&A, 292, 641

Jensen, E. L. N., Mathieu, R. D., \& Fuller, G. A. 1994, ApJ, 429, L29

Kenyon, S. J., \& Hartmann, L. 1995, ApJS, 101, 117 (KH95)

Kenyon, S. J., Brown, D. I., Tout, Ch. A., \& Berlind, P. 1998, AJ, 115,2491
Kraus, A. L., White, R. J., \& Hillenbrand, L. A. ApJ, 649, 306

Luhman, K. L., Whitney, B. A., Meade, M. R., Babler, B. L., Indebetouw, R., Bracker, S., \& Churchwell, E. B. 2006, ApJ, 647,1180

Luhman, K. L., Allen, P. R., Espaillat, C., Hartmann, L., \& Calvet, N. 2009, ApJ, submitted

Massarotti, A., Latham, D. W., Torres, G., Brown, R. A., \& Oppenheimer, B. D. 2005, AJ, 129, 2294

Mathis, J. S. 1990, ARA\&A, 28, 37

Mohanty, S., Jayawardhana, R., \& Basri, G. 2005, ApJ, 626, 498

Nguyen, D. C., Jayawardhana, R., van Kerkwijk, M. H.,

Brandeker, A., Scholz, A., \& Damjanov, I. 2009, ApJ, 695, 1648

Osterloh, M., \& Beckwith, S. V. W. 1995, ApJ, 439, 288

Quillen, A. C., Blackman, E. G., Frank, A., \& Varnière, P. 2004, ApJ, 612, L137

Rieke, G. H., et al. 2004, ApJS, 154, 25

Sargent, B., Forrest, W. J., D'Alessio, P., Li, A., Najita, J., Watson, D. M., Calvet, N., Furlan, E., et al. 2006, ApJ, 645, 395

Sargent, B. A., Forrest, W. J., Tayrien, C., McClure, M. K., Li, A., Basu, A. R., Manoj, P., Watson, D. M., et al. 2009, ApJ, 690, 1193

Sargent, B. A., Forrest, W. J., Tayrien, C., McClure, M. K., Watson, D. M., Sloan, G. C., Li, A., Manoj, P., et al. 2009, ApJS, 182, 477

Simon, M., \& Prato, L. 1995, ApJ, 450, 824

Skrutskie, M. F., Dutkevitch, D., Strom, S. E., Edwards, S., Strom, K. M., \& Shure, M. A. 1990, AJ, 99, 1187

Skrutskie, M. F., et al. 2006, AJ, 131, 1163

Sogawa, H., Koike, C., Chihara, H., Suto, H., Tachibana, S., Tsuchiyama, A., \& Kozasa, T. 2006, A\&A, 451, 357

Steffen, A. T., Mathieu, R. D., Lattanzi, M. G., Latham, D. W., Mazeh, T., Prato, L., Simon, M., Zinnecker, H., \& Loreggia, D. 2001, AJ, 122, 997

Strom, K. M., \& Strom, S. E. 1994, ApJ, 424, 237

Walter, F. M., \& Kuhi, L. V. 1981, ApJ, 250, 254

Weaver, W. B., \& Jones, G. 1992, ApJS, 78, 239

Werner, M. W. et al. 2004, ApJS, 154, 1

White, R. J., \& Basri, G. 2003, ApJ, 582, 1109

White, R. J., \& Ghez, A. M. 2001, ApJ, 556, 265

Woitas, J., Leinert, Ch., \& Köhler, R. 2001, A\&A, 376, 982

Wyatt, M. C., Dermott, S. F., Telesco, C. M., Fisher, R. S., Grogan, K., Holmes, E. K., \& Piña, R. K. 1999, ApJ, 527, 918 